\title{
Does Speeded Decision-Making Reveal Tacit Teleological Tendencies?
}

This manuscript has been anonymised for peer review

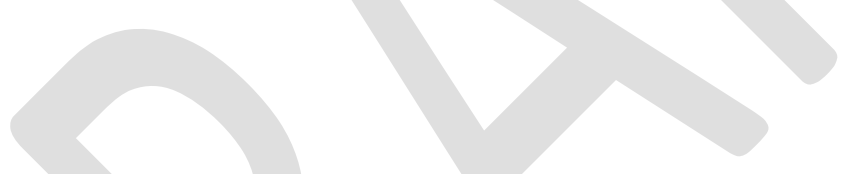

\section{Author Note}

This research did not receive any specific grant from funding agencies in the public, commercial, or not-for-profit sectors. We have no conflicts of interest to disclose. Data and code for analysis are stored on OSF and can be accessed at the following URL: https://osf.io/pw7vm/?view_only=ac540355ec00438e89b4fc841a386179. This link has been anonymised for the peer review process. 


\section{Abstract}

2 Previous research suggests that people implicitly believe that biological and nonbiological

3 natural entities exist to fulfil certain functions (i.e., people hold implicit teleological beliefs).

4 The standard experimental paradigm used to demonstrate this is to compare rates of

5 teleological acceptance in an un-speeded condition to acceptance in a speeded condition. As

6 speeded decision-making limits the opportunity to engage in reflective thought, increased

7 rates of teleological acceptance relative to the un-speeded condition are said to provide

8 evidence of implicit teleological beliefs. Across two large online studies, we show that due

9 to the exclusion criteria typically used in this paradigm, the included and excluded

10 participants vary systemically in important ways between conditions, and that increased rates

11 of teleological acceptance during speeded responding does not provide evidence of implicit

12 teleological beliefs. Rather, the difference between conditions can be explained by increased

13 acceptance of explanations which are objectively false. Furthermore, we show that a key

14 assumption underpinning the use of this paradigm - that accepting teleological explanations

15 should be effortless and rejecting them should require effort - is not supported by the data.

16 These results highlight not only a methodological issue, but also a theoretical issue in the

17 current literature. We discuss the implications of these findings in the context of existing

18 theoretical and empirical literature on teleological reasoning and dual-process theory more

19 generally.

20 Keywords: teleology, intentional stance, intuition, dual-process theory, implicit belief 


\section{Introduction}

Why are you reading this article? You might answer this question by referring to a series of events in your past which culminated in an interest in teleological reasoning or speeded decision-making tasks. Another possible answer is that you are reading this article to learn whether speeded decision-making can reveal tacit teleological tendencies. Whereas the former is a causal explanation about teleology, the latter is a teleological explanation about teleology. Put simply, teleological explanations are those in which something is explained with reference to a function, outcome, or goal (Hempel \& Oppenheim, 1948). Teleological reasoning is commonly used to explain the actions of intentional agents (as in the example above), the existence of human-made artefacts (e.g., "pens exist for writing"), and sometimes controversially (see Dennett, 2017), biological traits (e.g., "birds have wings in order to fly"). For intentional action and the existence of human-made artefacts, teleological reasoning is appropriate because both can be explained with reference to the intentions of an agent. If an agent creates a pen with the intention that this device will allow him or her to write, then that pen exists for writing. In the case of biological traits, teleological reasoning is appropriate not because of intentional action, but because of the consequence aetiology of natural selection (Dennett, 2017; Lombrozo \& Carey, 2006; Wright, 1976). That is, "birds have wings in order to fly", because the function of flight resulted in fitness advantages for those creatures with ancient wing-like structures. The function that wings serve, is the very reason for their current existence.

\subsection{Intention-Based Teleology}

Teleological reasoning is sometimes used to explain things which cannot be attributed to the actions of an intentional agent or the consequence aetiology of natural selection. According to Kelemen (1999a) all teleological reasoning is psychologically based in the application of an intentional stance (Dennett, 1987). This intention-based theory of teleology 
posits that from an early age, an intentional stance is applied beyond the domain of explaining intentional action. This is similar to the idea that the actual domain of a modular cognitive process extends beyond the module's proper domain (e.g., Atran \& Norenzayan, 2004; Boyer, 1994) ${ }^{1}$. Just as the application of an intentional stance to its proper domain results in a teleological explanation (e.g., "she crossed the road in order to get to the other side"), the application of this stance beyond its proper domain also results in a teleological explanation (e.g., "the computer crashed in order to annoy me"). As teleological beliefs are said to be based in the application of this early-developing predictive strategy, according to Kelemen, people often hold teleological beliefs even when the actions of an intentional agent are lacking. One domain of teleological reasoning that has received considerable attention, is the function-based explanation of biological and nonbiological natural entities (e.g., Kelemen, 1999c; Kelemen et al., 2013; Roberts et al., 2021; Willard et al., 2020).

\subsubsection{Non-Experimental Evidence for Intention-Based Teleology}

Teleological explanations such as "rivers flow downstream in order to get to the ocean", or "trees produce oxygen so that animals can breathe", tend to be accepted by children to a greater extent than adults (Kelemen, 1999b). However, under certain circumstances, adults also display high levels of acceptance for such teleological explanations. Rates of teleological acceptance in adults are positively correlated with anthropomorphism (Roberts et al., 2021; White et al., 2021; Willard et al., 2020; Willard \& Norenzayan, 2013) and belief in God (Kelemen \& Diyanni, 2005), suggesting that

\footnotetext{
${ }^{1}$ Although a discussion of modularity is beyond the scope of the current paper, there are many accessible sources on this topic (e.g., Grossi, 2014; Kurzban, 2010). Modularity theory posits that certain neural circuits are domain specific (Fodor, 1983). That is, cognitive modules selectively respond to certain stimuli in the environment. By the proper domain of a module, we mean the class of stimuli that a given cognitive process evolved "in response to" (e.g., a face detection module afforded fitness advantages to organisms with this neural circuit by allowing them to recognise faces of conspecifics). By the actual domain of a module, we mean all the stimuli to which a given cognitive process responds to (e.g., a face detection module may respond to masks because masks resemble faces).
} 
teleological reasoning is facilitated by notions of intentionality (whether to the natural world itself or to an intending designer). However, even non-religious individuals display evidence of teleological beliefs about biological and nonbiological natural entities (Järnefelt et al., 2015), supporting the view that such beliefs represent somewhat of a default mode of explanation.

Convergent non-experimental evidence also suggests that when people lack an alternative causal explanation, or that when access to the cognitive resources needed to inhibit intuitions is limited, people tend to be more accepting of teleological explanations about biological and non-biological natural entities. Evidence for this claim comes from studies showing increased teleological acceptance in 1) Alzheimer's patients relative to agematched controls (Lombrozo et al., 2007); 2) individuals with low levels of formal education relative to those with high levels of formal education (Casler \& Kelemen, 2008); and 3) children relative to adults (Kelemen, 1999b). Further non-experimental evidence comes from the negative relationship between performance on the Cognitive Reflection Test (CRT) and rates of acceptance for teleological explanations about biological and non-biological natural entities (Roberts et al., 2021; Zemla et al., 2012). Each item in the CRT is designed to elicit an intuitively appealing, yet incorrect response (e.g., "if you are running a race and you pass the person in second place, what place are you in?"). In this example, the intuitive response is "first place", but the correct response is "second place". As performance on the CRT requires an individual to inhibit the intuitive response, the negative relationship between CRT performance and rates of teleological acceptance has been taken as evidence that compared to low-scoring CRT participants, high-scoring CRT participants may simply be better at inhibiting their teleological intuitions. 


\subsubsection{Experimental Evidence for Intention-Based Teleology}

Experimental evidence for the claim that adults find teleological explanations about biological and nonbiological natural entities compelling, comes from the use of speeded vs. un-speeded decision-making tasks (Kelemen et al., 2013; Kelemen \& Rosset, 2009; Mills \& Frowley, 2014; Roberts et al., 2020). In this paradigm, participants are allocated to either a speeded or un-speeded condition and are asked to respond "true" or "false" (or "yes" or "no") to a series of teleological test statements and control statements. In the speeded condition, participants typically have either 3200ms (Kelemen et al., 2013; Kelemen \& Rosset, 2009; Mills \& Frowley, 2014; Roberts et al., 2020) or 5000ms (Kelemen \& Rosset, 2009; Mills \& Frowley, 2014; Rottman et al., 2017) to respond to each statement ${ }^{2}$. The logic behind using a speeded decision-making paradigm in the study of tacit teleological tendencies, is based in dual-process theories of reasoning (e.g., De Neys, 2014; Evans \& Stanovich, 2013;

Pennycook et al., 2015). As time-pressure limits the opportunity to reflect upon and inhibit an initial response, responses in the speeded condition are thought to provide an indication of pre-reflective belief (Kelemen et al., 2013). Consistent with the non-experimental evidence, studies employing this paradigm have reported significantly greater teleological acceptance in the speeded condition compared to the un-speeded condition.

\subsubsection{Problems with Speeded Decision-Making}

Despite the consistency between experimental and non-experimental evidence, a potential problem with the speeded vs. un-speeded paradigm, as it is commonly used, is that it may lead to selection bias. Inclusion criteria vary across studies, but participants in both conditions are typically required to respond correctly to a certain percentage of control statements, ranging from just $25 \%$ correct (Kelemen \& Rosset, 2009), to over $80 \%$ correct

\footnotetext{
${ }^{2}$ The instruction to "respond quickly" has also been used by Preston and Shin (2020). However, this study did not include an actual time limit.
} 
112 (Kelemen et al., 2013; Roberts et al., 2020). Additionally, participants in the speeded

113 condition are required to answer a certain percentage of test statements within the time limit,

114 ranging from $25 \%$ (Kelemen \& Rosset, 2009), to over 75\% (Kelemen et al., 2013; Roberts et

115 al., 2020). These inclusion criteria can result in a higher rate of exclusion in the speeded

116 condition compared to the un-speeded condition. For example, despite Roberts et al. (2020)

117 allocating 138 participants to either a speeded or un-speeded condition at a ratio of 1.5

118 (speeded): 1 (un-speeded), only 34 participants in the speeded condition and 54 in the un-

119 speeded condition met the inclusion criteria. The different rates of exclusion mean it is

120 possible that the participants vary systematically in certain ways between the two conditions.

121 As mentioned previously, there is a negative relationship between CRT performance

122 and rates of teleological acceptance in the absence of time pressure (Roberts et al., 2021;

123 Zemla et al., 2012). Given that each question on the CRT is designed to elicit an intuitively

124 appealing incorrect answer, performance on the CRT requires an individual to first inhibit

125 their intuitions, and to then reflect to produce the correct response. This leads to two

126 possibilities regarding the relationship between CRT performance and inclusion in the

127 decision-making task. If high-scoring CRT participants are more likely to pause and reflect

128 before providing an answer, this could lead to the systematic exclusion of these individuals in

129 the speeded condition if they fail to answer a sufficient percentage of test statements within

130 the time-limit. Due to the negative relationship between CRT performance and teleological

131 acceptance, excluding high-scoring CRT participants in the speeded condition could

132 artificially increase rates of teleological acceptance in this condition. However, it is also

133 conceivable that high-scoring CRT participants may be better able to answer quickly when

134 under time pressure to respond. As such, this could lead to the systematic exclusion of low-

135 scoring CRT participants in the speeded condition, thereby artificially decreasing rates of

136 teleological acceptance in this condition. Either of these possibilities would be problematic 
137 for the claim that speeded decision-making can provide an accurate assessment of tacit 138 teleological tendencies.

139 While the finding of increased teleological acceptance in the speeded compared to un140 speeded condition appears to be robust (Kelemen et al., 2013; Kelemen \& Rosset, 2009;;

141 Roberts et al., 2020; but see Liquin \& Lombrozo, 2018 - study 5), this alone is not evidence 142 of tacit teleological tendencies. The claim that this paradigm provides evidence of such tacit 143 tendencies, rests on the idea that the difference in responses between conditions is due to the 144 speeded condition limiting the opportunity to inhibit reflective thought. However, in this 145 paradigm, participants also respond to a series of objectively false and objectively true 146 control statements in either condition. As such, unless we are also willing to concede that 147 speeded decision-making provides evidence of implicit belief in things that are objectively 148 false (e.g., "people smoke cigarettes in order to get lung cancer"), or evidence of implicit 149 disbelief in things that are objectively true (e.g., "doctors prescribe antibiotics in order to treat 150 infections"), the effect of time pressure must be greater for teleological test statements than 151 for control statements.

152 At present, there is little evidence to suggest that the effect of time pressure is greater 153 for teleological test statements than for control statements. This is not necessarily due to the absence of such evidence in the data, but rather, due to the ways in which results have been reported, and to the slight differences in procedures across studies. For example, by

156 presenting groups of participants with statements on an overhead screen for varying durations

157 (un-speeded; moderately-speeded, 5000ms; and fast-speeded, 3200ms) and asking them to 158 respond by ticking boxes on an answer sheet, studies have found an effect of speed on 159 response accuracy for certain teleological statements (Kelemen \& Rosset, 2009; Mills \& 160 Frowley, 2014). When looking at control statement accuracy, Kelemen and Rosset (2009) 161 found that un-speeded participants were significantly less accurate than the other groups 
162 (Study One), although this effect failed to replicate in a second study (see also Mills \&

163 Frowley, 2014). However, given the manipulation of speed in these studies involved

164 participants being presented with statements on an overhead screen and responding by ticking

165 boxes on an answer sheet, it was possible for participants to respond after the statements had

166 disappeared from the screen. Therefore, it may not be so surprising that using this procedure,

167 participants were still highly accurate in responding to control statements in the speeded 168 conditions.

169 A more rigorous approach has been to use computerised tasks to limit not only the

170 duration of presentation, but also the response window. Using this approach, studies have

171 found an interaction between condition (un-speeded, speeded) and statement-type accuracy

172 (teleological, control). However, the way in which this interaction has been reported does not

173 necessarily provide evidence of tacit teleological tendencies. Using the speeded vs. un-

174 speeded paradigm to investigate the teleological beliefs of professional scientists, Kelemen et

175 al. (2013) found a larger mean difference between conditions for test statements (14\%

176 difference) than control statements (3\% difference). Similarly, using an undergraduate

177 sample, Roberts et al. (2020) found a larger mean difference between conditions for test

178 statements (18.9\% difference) than control statements (3.5\% difference). Such results have

179 also been replicated in a Chinese adult sample (Rottman et al., 2017), with a larger mean

180 difference between conditions for test statements (14\% difference) than control statements

181 (6\% difference). Examination of the descriptive statistics in these studies show that although

182 the mean differences were indeed larger for test statements than for control statements, since

183 control statements tend to be answered quite accurately, the variation in responses was also

184 larger for test statements than for control statements. As measures of effect size take account

185 of not only a difference in means, but also some component of error variance (e.g., Lakens,

186 2013), a greater mean difference in response accuracy between conditions for test statements 
187 than for control statements, does not necessarily equate to a larger effect size for the former than for the latter.

Finally, even if it can be shown that speeded decision-making has a greater effect on test statement accuracy than on control statement accuracy, this would still not necessarily

191 provide evidence of implicit teleological beliefs. As placing participants under cognitive load

192 has been shown to increase yea-saying (Knowles \& Condon, 1999), it is possible that speeded

193 decision-making merely increases the likelihood of accepting statements as true. As the

194 control statements used in the previously mentioned studies describe things which are

195 objectively true or false, when presented without a time-limit, they tend to be accepted at

196 close to ceiling and floor levels, respectively (Roberts et al., 2021). If speeded decision-

197 making does increase the likelihood of accepting statements as true, then comparing test

198 statement accuracy to the aggregate of all control statements may be problematic, as the true

199 control statements could reduce the difference in accuracy between conditions for control

statements as a whole. As teleological test statements describe beliefs that adults are hypothesised to inhibit, a stronger test of the hypothesis that speeded decision-making can uncover tacit teleological tendencies, would be to compare the effect of time pressure for teleological test statements relative to control statements that are objectively false. To provide evidence of tacit teleological tendencies, this paradigm must be able to demonstrate that the effect of time pressure for test statements is significantly greater than the effect of time pressure for false control statements.

\subsection{Current Studies}

In summary, there are three key issues in studies that have used the speeded vs. un-

209 speeded decision-making paradigm to explore implicit teleological beliefs. First, differing

210 inclusion criteria between experimental conditions may lead to the systematic exclusion of

211 certain individuals from one condition, but not the other. Second, reported interactions 
212 between speed and item type have not included enough information to ascertain whether

213 implicit teleological beliefs are truly driving the effects of interest. Third, comparing

214 responses to teleological test statements to the aggregate of all control statements (i.e., those

215 which are objectively false and those which are objectively true), may obscure differences in

216 accuracy between conditions. In the current two studies, we aimed to explore these issues

217 and determine whether the speeded decision-making paradigm provides evidence of tacit

218 teleological tendencies. These studies were approved by the [BLINDED] University Human

219 Research Ethics Committee (protocol number [BLINDED]), under the project titled "The

220 Intentional Stance and Teleological Endorsement”.

\section{Study One}

In Study One, we sought to explore the issues raised above using a large online

sample. First, we investigated whether the typical speeded vs. un-speeded paradigm results in selection bias. Due to the higher expected rate of exclusion in the speeded condition compared to the un-speeded condition, and due to the additional inclusion criterion and greater difficulty of the task in the former, we predicted that participants who met inclusion criteria in the speeded condition would differ significantly in their ability to inhibit intuitions relative to participants who did not meet inclusion criteria in this condition. Second, we investigated whether the speeded vs. un-speeded paradigm provides evidence of a dissociation between implicit and explicit teleological beliefs. If implicit teleological beliefs are truly driving the effects of interest, then the effect of time pressure should be greater for

232 teleological test statements than for control statements. Specifically, any increase in rates of 233 acceptance for teleological test statements under time pressure, should not be attributable to acceptance of statements that are objectively false. The preregistration for Study One can be accessed at https://osf.io/v83zm/?view_only=c9f406b1bf5d4f8e9cb5bf6a96301de1 . 


\subsubsection{Participants}

An international pool of 273 native English-speakers were recruited through the

online service, Prolific. This sample size was chosen based on previous research (Kelemen et

al., 2013) and taking into consideration the expected high rate of exclusion in the speeded

condition. Participants received payment of $£ 2.22$ for taking part in an online study that took approximately 15 minutes to complete. As we aimed to investigate whether inclusion in the speeded condition varied systematically with the tendency to inhibit intuitions, and as highly educated individuals tend to be both less teleological (Kelemen et al., 2013) and more analytical (Purcell et al., 2020), only individuals with formal educational qualifications no higher than an undergraduate university degree were eligible to participate. Before exclusion, the sample consisted of 92 atheists, 64 agnostics, 111 Christians $^{3}, 5$ Muslims, and 1 Jew. Ages ranged from 16 to $73(M=32.77, S D=13.37)$, with 141 females, 130 males, and two non-binary.

\subsubsection{Materials}

2.1.2.1. Teleological Beliefs Scale. Participants completed the Teleological Beliefs

Scale (TBS: Roberts et al., 2021) which measures teleological beliefs about biological and non-biological natural entities. The TBS contains 98 statements across six categories: biological teleological test $(n=14$; e.g., "Plants consume $\mathrm{CO} 2$ in order to reduce greenhouse gases"), non-biological teleological test ( $n=14$; e.g., "The Earth has a moon in order to control the tides"), false causal ( $n=25$; e.g., "Pebbles have rounded edges because they are little"), false teleological ( $n=10$; e.g., "Houses have doorbells in order to make dogs bark"),

\footnotetext{
${ }^{3}$ Participants were able to select "Christian" or "Catholic". Although Catholicism is a form of Christianity, we found in previous research that a substantial proportion of Catholics selected "Other" as their affiliation and then specified that they were Catholic in a free-response box. In the current sample, 72 selected Christian and 39 selected Catholic.
} 
true causal ( $n=25$; e.g., "Fireworks explode because gunpowder ignites when a fuse is lit"), and true teleological ( $n=10$; e.g., "Alarm clocks beep in order to wake people up"). In the current study, we collapsed both categories of test statements, both categories of false control statements, and both categories of true control statements. Participants responded "true" or "false" to each statement, and a total score for each category was calculated as the proportion of statements endorsed. The teleological test statements displayed good internal consistency $(\alpha=.894)$.

2.1.2.2. Attributions of Intentionality. To replicate findings of teleological beliefs about biological and non-biological natural entities being positively predicted by attributions of intentionality (e.g., Roberts et al., 2021; Willard et al., 2020; Willard \& Norenzayan, 2013), we included measures of anthropomorphism and religious belief.

2.1.2.2.1. Anthropomorphism. Participants completed the Anthropomorphism

Questionnaire (AQ: Neave et al., 2015), which contains 20 items measuring anthropomorphic experiences in childhood (e.g., "When I was a child, I held birthday parties for my favourite toys") and adulthood (e.g., "I sometimes feel that the sea can be angry"). Each item is scored on a scale from 0 (Not at all) to 6 (Very much so). A total score is obtained by summing all items, such that scores have a potential range of 0 to 120 . Internal consistency for the AQ was excellent $(\alpha=.939)$.

2.1.2.2.2. Religious Belief. Participants completed a reduced version of the Centrality of Religiosity Questionnaire (r-CRS: Huber \& Huber, 2012). The full version of the CRS contains 15 items balanced across five subscales: experience (e.g., "How often do you experience situations in which you have the feeling that God or something divine intervenes in your life?"), ideology (e.g., "To what extent do you believe that God or something divine exists?”), intellect (e.g., "How often do you think about religious issues?”), private practise (e.g., "How often do you pray?"), and public practise (e.g., "How often do you take part in 
283 religious services?"). As belief in God is not necessarily related to a desire to learn about

284 religious topics (intellect subscale) or attendance at religious services (public practise

285 subscale), we used a reduced version of the CRS which contained only the experience,

286 ideology, and private practise subscales (Roberts et al., 2021). Responses to questions about

287 the frequency of personal prayer were scored on a scale from 1 (Never) to 8 (Several times a

288 day) and then later recoded to a five-point scale (Huber \& Huber, 2012). The remaining

289 questions were scored on a scale from 1 (Never/Not at all) to 5 (Very often/Very much so).

290 A total score is calculated by taking the mean of all items. The r-CRS displayed excellent

291 internal consistency $(\alpha=.967)$.

2.1.2.3. Inhibition of Intuitions. To investigate whether the tendency to inhibit

293 intuitions negatively predicted scores on the TBS, and whether participants in the speeded condition were systematically excluded according to their tendency to engage in reflective thought, participants completed an extended version of the Cognitive Reflection Test (CRT). The original CRT contains three questions (e.g., "A bat and a ball cost $\$ 1.10$ in total. The bat costs $\$ 1.00$ more than the ball. How much does the ball cost?”: Frederick, 2005). In the current study, we used slightly re-worded versions of the original three questions (e.g., "The ages of Mark and Adam add up to 28 years. Mark is 20 years older than Adam. How many years old is Adam?"). Additionally, the extended version of the CRT contains four less math focused questions (e.g., “Emily’s father has three daughters. The first two are named April and May. What is the third daughter's name?": Thomson \& Oppenheimer, 2016). Each question is designed to elicit an intuitively appealing yet incorrect answer which must be

304 inhibited to produce the correct response. A total score on the CRT is calculated as the number of correct responses, such that scores have a possible range of 0 to 7 . The internal consistency of the CRT was acceptable $(\alpha=.725)$. 
307

308

309

310

311

312

313

314

315

316

317

318

321

322

323

\subsubsection{Procedure}

Participants were provided a link which redirected them to the study which was hosted on Gorilla.sc. After providing informed consent, participants were quasi-randomly assigned to either a speeded or un-speeded condition of the TBS to ensure a balanced design. In the un-speeded condition, participants were instructed to "read each statement carefully and respond according to what you believe", whereas in the speeded condition, participants were instructed to "read each statement and respond as quickly and as accurately as possible". Participants responded "true" or "false" to each item by pressing the $J$ or $F$ key, respectively. In the speeded condition, participants had $3200 \mathrm{~ms}$ in which to respond to each statement in the TBS. If they failed to respond within $3200 \mathrm{~ms}$, the next item appeared on the screen. In both conditions, once an item was first presented on the screen, there was a $500 \mathrm{~ms}$ window in which responses were not recorded. This was both to encourage participants to process the statement, and to ensure that participants in the speeded condition did not begin responding to a statement at $3200 \mathrm{~ms}$ but make their response once the following item was presented. After completing the TBS, participants completed the Anthropomorphism Questionnaire, reduced Centrality of Religiosity Scale, and extended Cognitive Reflection Test in a Latin square design.

Consistent with previous studies (Kelemen et al., 2013; Roberts et al., 2020), participants in either condition were excluded for failing to correctly respond to $80 \%$ of control statements, or for failing to respond to $75 \%$ of teleological test statements within the time-limit in the speeded condition. In the un-speeded condition, 11 participants failed to meet the inclusion criterion $(n=100)$, whereas in the speeded condition, 62 participants failed to meet the inclusion criteria $(n=100)$. The difference in inclusion between conditions was statistically significant $\chi^{2}(1)=27.00, p<.001$. 


\subsection{Results}

332

333

334

335

336

337

338

339

340

341

342

\subsubsection{Correlations Between Predictors and Responses on the Teleological Beliefs Scale}

We first explored the relationships between acceptance of teleological explanations about biological and non-biological natural entities, accuracy in responding to control statements that were objectively true or objectively false, and anthropomorphism (AQ), belief in God (r-CRS), and inhibition of intuitions (CRT). As shown in Table 1, before participant exclusion, anthropomorphism and belief in God displayed weak but significant positive relationships with teleological acceptance, whereas inhibition of intuitions displayed a moderate-to-strong significant negative relationship with teleological acceptance. Anthropomorphism and belief in God were weakly and negatively related to false control statement accuracy, whereas inhibition of intuitions had a moderate-to-strong positive relationship with false control statement accuracy. 


\section{Table 1}

Correlations Between Teleology and Predictor Variables in Study One Prior to Exclusion

\begin{tabular}{|c|c|c|c|c|c|c|}
\hline & Teleology & False & True & $\mathrm{AQ}$ & r-CRS & CRT \\
\hline \multirow[t]{2}{*}{ Teleology } & - & & & & & \\
\hline & - & & & & & \\
\hline \multirow[t]{2}{*}{ False Control } & $-.587 * * *$ & - & & & & \\
\hline & {$[-.66,-.50]$} & - & & & & \\
\hline \multirow[t]{2}{*}{ True Control } & $-.247 * * *$ & $.556 * * *$ & - & & & \\
\hline & {$[-.36,-.13]$} & {$[.47, .63]$} & - & & & \\
\hline \multirow[t]{2}{*}{ AQ } & $.181 * *$ & $-.123 *$ & -.026 & & & \\
\hline & {$[.06, .29]$} & {$[-.24,-.01]$} & {$[-.15, .09]$} & 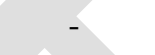 & & \\
\hline \multirow[t]{2}{*}{ r-CRS } & $.213 * * *$ & $-.197 * *$ & $-.136^{*}$ & .095 & - & \\
\hline & {$[.10, .32]$} & {$[-.31,-.08]$} & {$[-.25,-.02]$} & {$[-.02, .21]$} & - & \\
\hline \multirow[t]{2}{*}{ CRT } & $-.414 * * *$ & $.406 * * *$ & $.170 * *$ & -.105 & $-.181 * *$ & - \\
\hline & {$[-.51,-.31]$} & {$[.30, .50]$} & {$[.05, .28]$} & {$[-.22, .01]$} & {$[-.29,-.06]$} & - \\
\hline
\end{tabular}

Note. Pearson's $r$ correlations with $95 \%$ confidence intervals [L, U]. $N=273$ for all cells. "Teleology" represents agreement with test statements, whereas "False" and "True" represent accuracy for each category of control statements. AQ = anthropomorphism, $\mathrm{r}-\mathrm{CRS}=$ belief in God, CRT = inhibition of intuitions. $* p<.05, * * p<.01, * * * p<.001$.

\subsubsection{Inclusion and Exclusion}

344 Scores on the CRT displayed moderate to strong significant relationships with both

345 teleological acceptance and accuracy for false control statements. As accuracy on control

346 statements was one of inclusion criteria, next we explored whether scores on the predictor

347 variables differed between those who met and those who did not meet the inclusion criteria.

348 As shown in Table 2, there was no significant difference in anthropomorphism between

349 included and excluded participants, $t(271)=-0.62, p=.537, d=0.085$. However, compared

350 to excluded participants, those who met inclusion criteria expressed less belief in God, $t(271)$

$351=-2.44, p=.015, d=0.334$, and were better able to inhibit their intuitions, $t(271)=4.75, p<$ $352 \quad .001, d=0.650$. 


\section{Table 2}

Comparison of Scores on Predictor Variables for Included and Excluded Participants in Study One

\begin{tabular}{lccc}
\hline & Included & Excluded & Difference \\
\hline Anthropomorphism (AQ) & $30.87(1.82)$ & $33.03(2.96)$ & $2.16(3.50),[-4.73,9.05]$ \\
Belief in God (r-CRS) & $2.18(0.08)$ & $2.57(0.15)$ & $0.39(0.16),[0.08,0.71]^{*}$ \\
Inhibition (CRT) & $3.64(0.13)$ & $2.41(0.24)$ & $-1.22(0.26),[-1.73,-0.72]^{* * *}$
\end{tabular}

Note. Means are shown with standard errors in parentheses. $95 \%$ confidence intervals $[\mathrm{L}, \mathrm{U}]$ are shown for mean differences. Included $n=200$. Excluded $n=73$.

$* p<.05, * * * p<.001$.

\subsubsection{The Effect of Speed on Teleology}

To investigate whether speeded decision-making reveals tacit teleological tendencies, using only participants who met the inclusion criteria, we compared responses to the teleological test statements and control statements between the speeded and un-speeded conditions. If teleological beliefs about biological and non-biological natural entities are a “developmentally persistent cognitive default" (Kelemen et al., 2013, p. 1075), and if speeded decision-making can be used as a method for uncovering such beliefs, then time pressure should have a significantly greater effect for teleological test statements than for control statements. To allow for a meaningful comparison of statement types, responses to teleological test statements and false control statements were reverse-coded, such that higher scores represented greater accuracy rather than greater acceptance. We conducted a 2 (condition: speeded, un-speeded) x (3) (statement-type: teleological test, false control, true control) ANOVA with accuracy as the dependent variable.

The effects of condition, $F(1,198)=58.49, p<.001, \eta \rho^{2}=.228$, and statement-type, $F(1,396)=606.34, p<.001, \eta \rho^{2}=.754$, were both significant, but as shown in Figure 1, were qualified by a significant condition by statement-type interaction, $F(1,396)=13.03, p<$ $.001, \eta \rho^{2}=.062$. To explore this interaction, three contrasts were performed in which we compared the difference between conditions on test statement accuracy relative to overall 
371 control statement accuracy (contrast 1), test statement accuracy relative to false control

372 statement accuracy (contrast 2), and test statement accuracy relative to true control statement

373 accuracy (contrast 3). Mean accuracies are shown in Table 3.

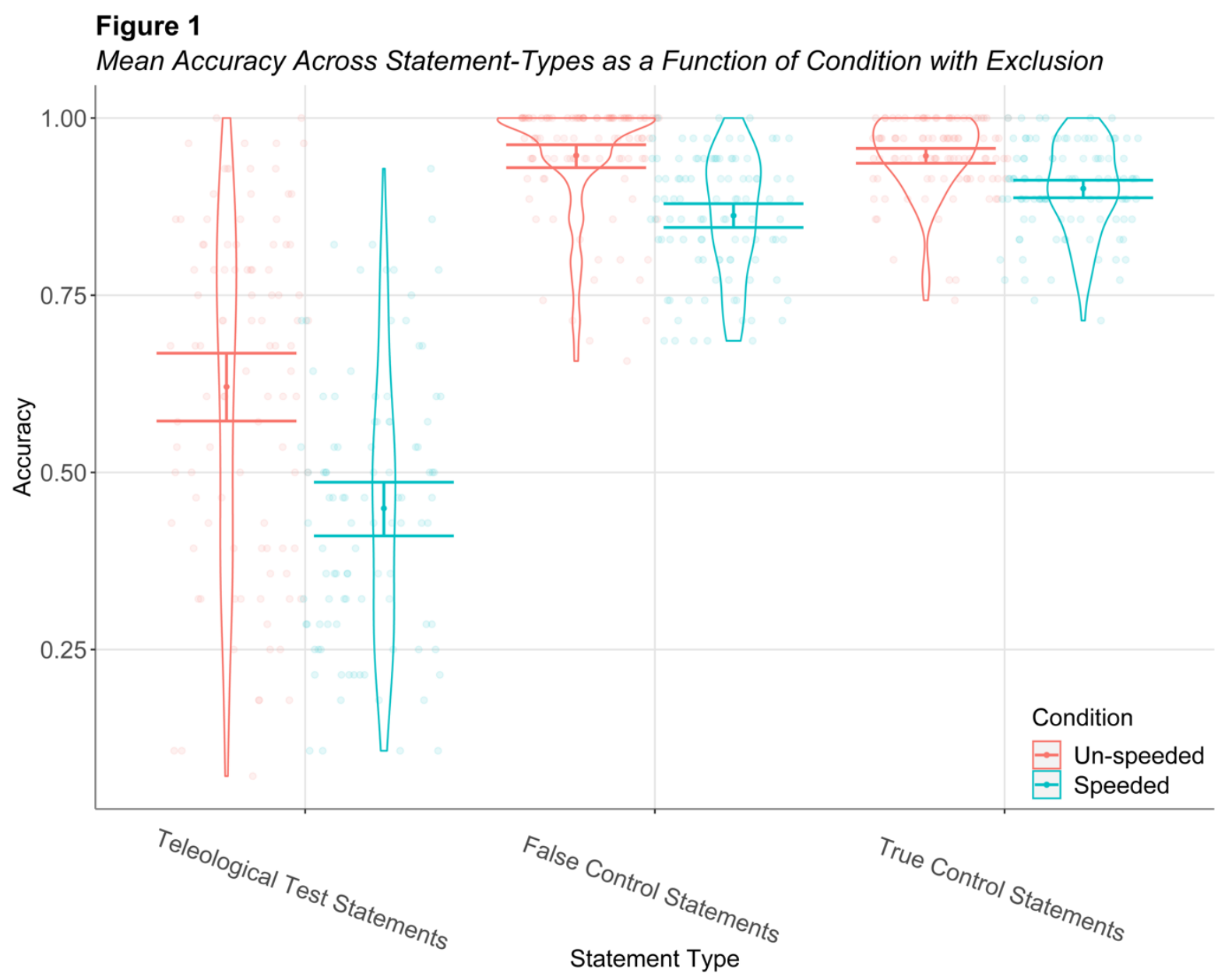




\section{Table 3}

Accuracy for Statement Types as a Function of Condition after Participant Exclusion in Study One

\begin{tabular}{lcccc}
\hline & Teleological Test & Control Aggregate & False Control & True Control \\
\hline Un-Speeded & $0.62(0.24)$ & $0.95(0.05)$ & $0.95(0.08)$ & $0.95(0.05)$ \\
Speeded & $0.45(0.19)$ & $0.88(0.05)$ & $0.86(0.09)$ & $0.90(0.06)$ \\
Difference & $0.17[0.11,0.23]$ & $0.07[0.05,0.08]$ & $0.08[0.06,0.11]$ & $0.05[0.03,0.06]$
\end{tabular}

Note. Means are shown with standard deviations in parentheses. 95\% confidence intervals are shown [L, U] for mean differences between conditions. revealed tacit teleological tendencies, the effect of time pressure on control statement accuracy in aggregate, $t(198)=9.19, p<.001, d=1.300$, was stronger than the effect of time pressure on teleological test statement accuracy, $t(198)=5.57, p<.001, d=0.788$. This difference was statistically significant, $F(1,198)=14.18, p<.001, \eta \rho^{2}=.067$. Similarly, the effect of time pressure on false control statement accuracy, $t(198)=7.27, p<.001, d=1.028$, was stronger than the effect of time pressure on teleological test statement accuracy $(F(1$, 198) $\left.=10.99, p=.001, \eta \rho^{2}=.053\right)$. However, the effect of time pressure on teleological test statement accuracy was stronger than the effect of time pressure on true control statement accuracy, $t(198)=5.50, p<.001, d=0.778$, and this difference was statistically significant $\left(F(1,198)=15.62, p<.001, \eta \rho^{2}=.073\right)$. These results suggest that with the inclusion criteria of $>80 \%$ accuracy on control statements and $>75 \%$ of test statements answered within the time limit, speeded responding has a greater effect on control statement accuracy than on teleological test statement accuracy. This effect was driven by decreased accuracy for false control statements in the speeded condition rather than by changes in teleological endorsement. 


\subsubsection{Response Latencies}

Although the preceding results do not necessarily show that teleological tendencies do not exist, they do demonstrate a problem with the speeded decision-making paradigm in the study of teleological tendencies. In an exploratory analysis, we investigated response latencies for correctly rejected and incorrectly accepted teleological test statements. If teleological beliefs about biological and nonbiological natural entities are a developmentally persistent cognitive default, then people should be faster to accept than to reject such explanations. Indeed, the very basis of speeded decision-making as a method of demonstrating such tendencies, rests on the idea that rejecting such explanations requires cognitive effort, and hence, time.

Using only the un-speeded condition, mean latencies were calculated separately for correctly rejected and incorrectly accepted teleological test statements for each participant. Standardised scores were computed based on these means, resulting in the exclusion of one participant with a Z-score of 15.56. All other mean z-scores were within a range of -0.42 to 1.16. Next, standardised scores were calculated for individual trials. Latencies for trials with a corresponding z-score greater than $+/-3$ were replaced with the mean latency of either correctly or incorrectly answered trials depending on the response. Two participants who responded correctly to every statement were excluded, leaving 97 participants. A paired ttest revealed no significant difference in response latencies between correctly rejected $(M=$ 4456.34, $S D=1910.15)$ and incorrectly accepted teleological test statements $(M=4353.89$, $S D=2440.54), t(96)=0.49, p=.628$. To better understand this null finding, we ran a Bayesian paired t-test using an uninformative prior. This test provided moderate support for the null hypothesis of no difference between conditions $\left(\mathrm{BF}_{10}=0.172\right)$.

A possible objection to the previous analysis is that perhaps certain teleological test statements tend to be answered correctly, whereas certain others tend to be answered 
415 incorrectly. If response latencies differed systematically across statements, then the previous

416 null result could be due to statement-specific variation in latencies. That is, perhaps people

417 are faster to accept than to reject teleological statements about biological and nonbiological

418 natural entities in general, but perhaps the statements in the Teleological Beliefs Scale that

419 people tend to accept, also happen to be those that take longer to process regardless of the

420 response. To rule out this alternative explanation, we repeated the previous paired t-test, but

421 restricted the analysis to statements with a mean accuracy of between 0.4 and 0.6. As before,

422 the paired t-test revealed no significant difference in response latencies between correctly

423 rejected $(M=4800.63, S D=2550.46)$ and incorrectly accepted teleological test statements

$424(M=5048.01, S D=3601.71), t(86)=-0.64, p=.524$. A Bayesian paired t-test using an

425 uninformative prior provided strong support for the null hypothesis of no difference between

426 conditions $\left(\mathrm{BF}_{10}=0.077\right)^{4}$.

427

428

430

431

432

433

434

435

436

\subsection{Discussion}

Study One replicated the previously reported relationships between teleological beliefs about biological and nonbiological natural entities and anthropomorphism (Roberts et al., 2021; Willard et al., 2020; Willard \& Norenzayan, 2013), belief in God (Kelemen \& Diyanni, 2005), inhibition of intuitions (Zemla et al., 2012). Specifically, teleological beliefs were positively predicted by anthropomorphism and belief in God, and negatively predicted by the tendency to inhibit intuitions. The negative relationship between inhibition of intuitions and teleological beliefs is particularly important, as this has been taken as evidence that teleological beliefs can be conceptualised within a dual-process framework (Roberts et al., 2021; Zemla et al., 2012). According to this view, teleological beliefs about biological and non-biological natural entities are intuitively appealing, and to not express such beliefs,

\footnotetext{
${ }^{4}$ Without the replacement of outliers, the interpretation of response latency comparisons did not change. All statements, $t(97)=-0.86, p=.390$; only statements endorsed at moderate rates, $t(97)=0.36, p=.723$.
} 
438 effortful thought must be engaged (Kelemen et al., 2013; Kelemen \& Rosset, 2009).

439 However, we also found that the tendency to inhibit intuitions was positively related to

440 accuracy in responding to control statements that were objectively false. Given the inclusion

441 criterion of responding to over $80 \%$ of control statements correctly, the bivariate relationships

442 highlighted the possibility that participants could be systematically excluded from the task

443 according to their ability to inhibit intuitions.

444 As expected, the rate of exclusion in the speeded condition was significantly higher

445 than in the un-speeded condition. Collapsed across conditions, participants who met the

446 inclusion criteria were significantly less religious and significantly better at inhibiting their

447 intuitions. This is problematic, as it demonstrates the systematic exclusion of certain

448 individuals in one condition, but not in the other. The direction of this relationship was such

449 that individuals who tend to express more teleological beliefs are systematically excluded

450 from the speeded condition, thereby potentially resulting in decreased teleological acceptance

451 in this condition. In other words, the exclusion of these individuals from the speeded

452 condition may provide an overly conservative estimate of tacit teleological tendencies.

453 However, more fundamentally problematic for claims of speeded decision-making

454 providing evidence for tacit teleological tendencies, was the effect of time pressure on

455 responses to teleological test statements and control statements. The results of Study One

456 showed that the effect of time pressure for teleological test statements, was weaker than the

457 effect of time pressure for control statements on average. Contrast testing revealed that this

458 difference was driven by reduced accuracy when responding to objectively false control

459 statements in the speeded condition. Rather than time pressure uncovering tacit teleological

460 tendencies, this suggests that time pressure increases acceptance of things which are false; of

461 which, it could be argued, teleological explanations about biological and nonbiological

462 natural entities are a part. 
Although the effect of time pressure on control statement accuracy relative to teleological test statement accuracy highlights a methodological issue, examination of response latencies for correctly rejected and incorrectly accepted teleological test statements suggests a problem with theory. The basis for using speeded decision-making to uncover tacit teleological tendencies, is that additional processing, and hence, time, should be required to inhibit the expression of such beliefs. Contrary to these predictions, we found that response latencies for correctly rejected and incorrectly accepted teleological test statements did not differ. Even when restricting our analysis to those statements with a mean acceptance of between $40 \%$ and $60 \%$, there was no evidence of a difference in response latencies between correct and incorrect responses on these items. In both cases, Bayesian analyses suggested there was moderate to strong evidence in favour of the null hypothesis of no difference in latencies between correct and incorrect responses.

\section{Study Two}

The findings of systematic exclusion in the speeded condition and the comparison of

477 effect sizes rather than absolute differences in means between conditions have not previously 478 been reported in the literature. In Study Two, we sought to replicate the results of Study One 479 using an undergraduate sample. First, we predicted that participants who met inclusion 480 criteria would be significantly better at inhibiting their intuitions compared to participants 481 who did not meet inclusion criteria. Second, we predicted that the effect of time pressure 482 would be significantly greater for false control statements than for teleological test

483 statements. The preregistration for Study Two can be accessed at 484 https://osf.io/zprxs/?view_only=e1e937fee3bf49d582f30294c8b1b664. 


\subsection{Methods}

\subsubsection{Participants}

Two-hundred and fifty first-year undergraduate psychology students from

[BLINDED] University in [BLINDED] participated in the study in exchange for course

credit. This sample size was chosen to be consistent with Study One. Before exclusion, the sample consisted of 82 agnostics, 72 atheists, 83 Christians, 8 Muslims, 4 Jews, and 1 “other". Ages ranged from 16 to $52(M=21.75, S D=7.17)$, with 183 females and 67 males.

\subsubsection{Materials}

The materials were identical to Study One.

\subsubsection{Procedure}

The procedure was identical to Study One. In the un-speeded condition, 6 participants failed to meet the inclusion criterion, whereas in the speeded condition, 76 participants failed to meet inclusion criteria. This resulted in a final sample of $n=119$ in the un-speeded condition, and $n=49$ in the speeded condition. The difference in rates of inclusion between conditions was statistically significant $\chi^{2}(1)=88.90, p<.001$.

\subsection{Results}

\subsubsection{Correlations Between Predictors and Responses on the TBS}

As shown in Table 4, before participant exclusion, anthropomorphism (AQ) was

positively correlated with teleological acceptance, whereas inhibition of intuitions (CRT) was negatively correlated with teleological acceptance. In contrast to Study One, the relationship between belief in God (r-CRS) and teleological acceptance was non-significant. statements, and inhibition of intuitions was positively related to accuracy for false control statements. Inhibition of intuitions also displayed a weak positive relationship with accuracy for true control statements. 


\section{Table 4}

Correlations Between Teleology and Predictor Variables in Study Two Prior to Exclusion

\begin{tabular}{|c|c|c|c|c|c|c|}
\hline & Teleology & False & True & $\mathrm{AQ}$ & r-CRS & CRT \\
\hline Teleology & - & & & & & \\
\hline False & $\begin{array}{c}-.624 * * * \\
{[-.69,-.54]}\end{array}$ & - & & & & \\
\hline True & $\begin{array}{c}-.159 * \\
{[-.28,-.04]}\end{array}$ & $\begin{array}{l}.439 * * * \\
{[.33, .53]}\end{array}$ & - & & & \\
\hline AQ & $\begin{array}{c}.188 * * \\
{[.07, .31]}\end{array}$ & $\begin{array}{c}-.211 * * * \\
{[-.33,-.09]}\end{array}$ & $\begin{array}{c}.065 \\
{[-.06, .19]}\end{array}$ & & & \\
\hline r-CRS & $\begin{array}{c}.075 \\
{[-.05, .20]}\end{array}$ & $\begin{array}{c}-.177 * * \\
{[-.30,-.06]}\end{array}$ & $\begin{array}{c}-.038 \\
{[-.16, .09]}\end{array}$ & $\begin{array}{l}.273 * * * \\
{[.15, .38]}\end{array}$ & - & \\
\hline CRT & $\begin{array}{c}-.219 * * * \\
{[-.33,-.10]}\end{array}$ & $\begin{array}{c}.265^{* * *} \\
{[.15, .38]}\end{array}$ & $\begin{array}{c}.167 * * \\
{[.04, .29]}\end{array}$ & $\begin{array}{c}-.203 * * \\
{[-.32, .08]}\end{array}$ & $\begin{array}{c}.140 * \\
{[-.26,-.02]}\end{array}$ & - \\
\hline
\end{tabular}

Note. Pearson's $r$ correlations with $95 \%$ confidence intervals [L, U]. $N=250$ for all cells. "Teleology" represents agreement with test statements, whereas "False" and "True" represent accuracy for each category of control statements.

$* p<.05, * * p<.01, * * * p<.001$.

\subsubsection{Inclusion and Exclusion}

The bivariate correlations conceptually replicated the key findings from Study One.

512 Performance on the CRT was significantly related not only to teleological acceptance, but to

513 accuracy for control statements. Given the control statement accuracy inclusion criterion, we

514 sought to replicate the finding that inhibition of intuitions varied systematically with

515 inclusion status. As shown in Table 5, included and excluded participants did not differ

516 significantly on anthropomorphism, $t(248)=1.83, p=.068, d=0.247$, or, in contrast to

517 Study One, belief in God, $t(248)=1.43, p=.155, d=0.192$. However, consistent with Study

518 One, compared to participants who failed to meet the inclusion criteria, those who met the

519 inclusion criteria were significantly better at inhibiting their intuitions, $t(248)=-3.45, p<$ $.001, d=0.465$. 


\section{Table 5}

Comparison of Scores on Predictor Variables for Included and Excluded Participants in Study Two

\begin{tabular}{lccc}
\hline & Included & Excluded & Difference \\
\hline Anthropomorphism (AQ) & $30.48(1.81)$ & $36.57(2.96)$ & $6.09[-0.45,12.63]$ \\
Belief in God (r-CRS) & $2.38(0.08)$ & $2.60(0.13)$ & $0.21[-0.08,0.50]$ \\
Inhibition of intuitions (CRT) & $3.65(0.13)$ & $2.85(0.19)$ & $-0.80[-1.25,-0.34]^{* *}$
\end{tabular}

Note. Means are shown with standard errors in parentheses. 95\% confidence intervals [L, U] are shown for mean differences. Included $n=168$. Excluded $n=82$.

$* * p<.01$.

521

522

523

524

525

526

527

528

529

530

\subsubsection{The Effect of Speed on Teleology}

Using only participants who met the inclusion criteria, we compared responses to the teleological test statements and control statements between the speeded and un-speeded condition. We conducted a 2 (condition: speeded, un-speeded) x (3) (statement-type:

teleological test, false control, true control) ANOVA with accuracy as the dependent variable.

The effects of condition, $F(1,166)=58.57, p<.001, \eta \rho^{2}=.261$, and statement-type, $F(1$, $332)=401.76, p<.001, \eta \rho^{2}=.708$, were both highly significant, but as shown in Figure 2, were qualified by a significant condition by statement-type interaction, $F(1,332)=16.38, p<$ $.001, \eta \rho^{2}=.090$. To explore this interaction, the same three contrasts were performed as in Study One. Mean accuracies are shown in Table 6.

\section{Table 6}

Accuracy for Statement Types as a Function of Condition after Participant Exclusion in Study Two

\begin{tabular}{lcccc}
\hline & Teleological Test & Control Aggregate & False Control & True Control \\
\hline Un-Speeded & $0.61(0.23)$ & $0.93(0.05)$ & $0.95(0.06)$ & $0.91(0.08)$ \\
Speeded & $0.40(0.19)$ & $0.87(0.05)$ & $0.85(0.09)$ & $0.89(0.07)$ \\
Difference & $0.21[0.13,0.28]$ & $0.06[0.05,0.08]$ & $0.11[0.08,0.13]$ & $0.02[-0.001,0.05]$
\end{tabular}

Note. Means are shown with standard deviations in parentheses. 95\% confidence intervals are shown [L, U] for mean differences between conditions. 


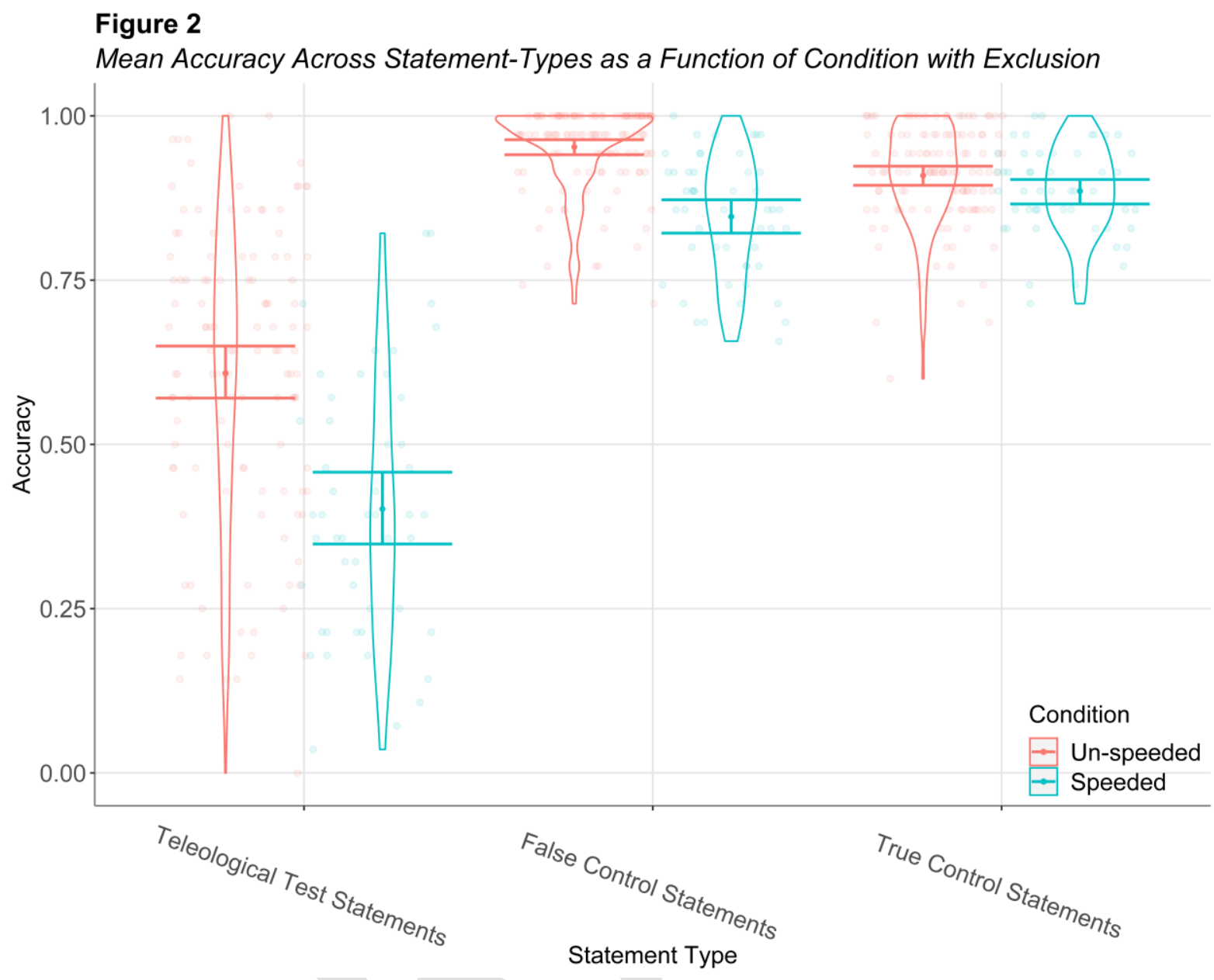

Contrary to what would be expected if the speeded decision-making paradigm

532 revealed tacit teleological tendencies, the effect of time pressure on control statement

533 accuracy in aggregate, $t(166)=5.58, p<.001, d=1.286$, was stronger than the effect of time

534 pressure on teleological test statement accuracy, $t(166)=5.60, p<.001, d=0.951$. This comparison was statistically significant, $F(1,166)=15.55, p<.001, \eta \rho^{2}=.086$. Similarly, the effect of time pressure on false control statement accuracy, $t(166)=8.65, p<.001, d=$

537 1.467, was stronger than the effect of time pressure on teleological test statement accuracy, $538 F(1,166)=8.96, p=.003, \eta \rho^{2}=.051$. However, the effect of time pressure on teleological test statement accuracy was stronger than the effect of time pressure on true control statement 
540 accuracy, $t(166)=1.86, p=.065, d=0.315$, and this comparison was statistically significant,

$541 \quad F(1,166)=20.66, p<.001, \eta \rho^{2}=.111$.

542

543

544

545

546

547

548

549

550

551

552

553

554

555

556

557

558

559

560

561

562

563

\subsubsection{Response Latencies}

We next investigated response latencies for correctly rejected and incorrectly accepted teleological test statements. Following the same procedure as Study One, using only the unspeeded condition $(N=119)$, mean latencies were calculated separately for correctly and incorrectly answered teleological test statements for each participant. Standardised scores were computed based on these means, resulting the exclusion of four participants with zscores greater than 4.25. All other z-scores were within a range of -0.75 to 2.13 . Next, standardised scores were calculated for individual trials. Latencies for trials with a corresponding $\mathrm{z}$-score greater than $+/-3$ were replaced with the mean latency of either correctly or incorrectly answered trials. A further four participants were excluded for not having both correct and incorrect responses to the test statements, leaving 111 participants. A paired t-test revealed no significant difference in response latencies between correctly rejected $(M=4812.52, S D=1919.15)$ and incorrectly accepted teleological test statements $(M=4898.53, S D=2386.45), t(110)=-0.43, p=.668$. A Bayesian paired t-test using an uninformative prior provided strong support for the null hypothesis of no difference between conditions $\left(\mathrm{BF}_{10}=0.077\right)$.

As a more conservative test of the difference in response latencies between correctly rejected and incorrectly accepted teleological test statements, we restricted our analysis to statements with a mean accuracy of between $40 \%$ and $60 \%$. As before, the paired t-test revealed no significant difference in response latencies between correctly rejected $(M=$ $4786.37, S D=2559.61)$ and incorrectly accepted teleological test statements $(M=5110.01$, $S D=3108.70), t(98)=-1.06, p=.293$. A Bayesian paired t-test using an uninformative 
564 prior provided strong support for the null hypothesis of no difference between conditions

$565 \quad\left(\mathrm{BF}_{10}=0.057\right)^{5}$.

566

567

568

569

570

571

572

573

574

575

576

577

578

579

580

581

582

583

584

\subsection{Discussion}

Using an undergraduate sample, Study Two replicated the key findings from Study

One. Acceptance of teleological statements about biological and non-biological natural entities was positively related to anthropomorphism and negatively related to inhibition of intuitions. Importantly, the tendency to inhibit intuitions was also positively related to accuracy for false control statements. Rates of exclusion differed significantly between conditions, and participants who met the inclusion criteria were significantly better at inhibiting their intuitions compared to those who did not meet the inclusion criteria. In combination, these results suggest that individuals who are worse at inhibiting their intuitions are disproportionately excluded from the speeded condition.

Study Two also replicated the finding of speeded decision-making having a significantly greater effect on false control statement accuracy than on teleological test statement accuracy. These findings suggest that the previously reported increase in teleological acceptance in a speeded condition relative to an un-speeded condition (e.g., Kelemen et al., 2013; Kelemen \& Rosset, 2009; Roberts et al., 2020), may be due to increased acceptance of explanations which are objectively false, rather than tacit teleological tendencies. Finally, replicating the findings from Study One, we found that response latencies for correctly rejected and incorrectly accepted teleological test statements did not differ.

\footnotetext{
${ }^{5}$ Without the replacement of outliers, the interpretation of response latency comparisons did not change. All statements, $t(115)=-0.06, p=.948$; only statements endorsed at moderate rates, $t(102)=0.55, p=.583$.
} 


\section{General Discussion}

The central claim of an intention-based theory of teleology, is that due to an early

developing understanding that intentional agents have purposes, people have a developmentally persistent tendency to explain the world in terms of purpose and function (Kelemen, 1999a). While there is convergent non-experimental evidence to support this claim (e.g., Casler \& Kelemen, 2008; Lombrozo et al., 2007; Zemla et al., 2012), experimental evidence comes from comparing rates of teleological acceptance in speeded and un-speeded conditions (e.g., Kelemen et al., 2013; Mills \& Frowley, 2014; Roberts et al., 2020). Studies employing this paradigm have reported that by limiting the time available to reflect upon beliefs, participants show increased teleological acceptance relative to an unspeeded condition. This difference in teleological acceptance between conditions has been taken as evidence that adults have tacit teleological tendencies. teleological beliefs about biological and non-biological natural entities were positively related to anthropomorphism and, in Study One, belief in God. Importantly, in both studies we found that teleological beliefs were negatively related to the tendency to inhibit intuitions. This is consistent with previous findings (Roberts et al., 2021; Zemla et al., 2012), and supports the view that an intention-based theory of teleology can be conceptualised within a dual-process framework. This dual-process perspective suggests that teleological beliefs about biological and non-biological natural entities represent a "developmentally persistent cognitive default" (Kelemen et al., 2013, p. 1075). Although teleological beliefs about biological and non-biological natural entities may be less frequently expressed by adults than by children, according to this view, such beliefs continue to have an intuitive appeal throughout adulthood. 
Using both an online community sample and an undergraduate psychology student

611 sample, the current studies identified several problems with the use of the speeded vs. un-

612 speeded paradigm in the study of teleological beliefs. As this paradigm necessitates the

613 exclusion of participants who respond inaccurately to control statements or fail to respond to

614 a sufficient proportion of test statements within the time limit, this leads to different rates of

615 exclusion between the conditions (Roberts et al., 2020). Exclusion was also found to vary

616 systematically with the tendency to inhibit intuitions, with participants scoring lower on the

617 CRT more likely to be excluded. Due to the significantly higher rate of participant exclusion

618 in the speeded condition than in the un-speeded condition, participants scoring lower on the

619 CRT were disproportionately excluded in the speeded condition, but not in the un-speeded

620 condition. As scores on the CRT were negatively related to rates of teleological acceptance, systematically excluding low-scoring CRT participants from the speeded condition means this paradigm may artificially decrease the mean rate of teleological acceptance in the speeded condition following participant exclusion. As a result, this paradigm may provide an overly conservative estimate of teleological acceptance in the speeded condition.

More problematic for research employing this paradigm in the study of teleological beliefs, is that the effect of time pressure on teleological acceptance, which has previously been taken as evidence of tacit teleological tendencies (e.g., Kelemen et al., 2013; Kelemen \& Rosset, 2009; Mills \& Frowley, 2014; Roberts et al., 2020), was weaker than the effect of time pressure on control statement (in)accuracy. That is, although the mean difference in accuracy between conditions was larger for teleological test statements than for control statements, the variation in accuracy for teleological test statements was also larger than for control statements. In both studies, the effect of time pressure for control statement inaccuracy was driven by responses to control statements that were objectively false. Rather 
that speeded decision-making increases rates of acceptance for statements that are objectively false. Therefore, unless we are willing to concede that people implicitly believe that "post-it notes are sticky because they are yellow", or that "people smoke cigarettes in order to get lung cancer", it is problematic to claim that this paradigm provides evidence of tacit teleological tendencies.

While our results show that the speeded vs. un-speeded paradigm does not provide evidence for a dissociation of implicit and explicit teleological beliefs, in isolation, this would be considered a methodological issue, but not necessarily a theoretical one. That is, an intention-based theory of teleology suggests that teleological beliefs about biological and

644 non-biological natural entities are a "developmentally persistent cognitive default" (Kelemen 645 et al., 2013, p. 1075), but this theory does not rest on the claim that a speeded decisionmaking task can uncover such tendencies. However, the appropriateness of speeded

647 decision-making in dissociating implicit and explicit teleological beliefs, is based on two theoretical assumptions. First, teleological beliefs are conceptualised as existing within a dual-process framework (Kelemen et al., 2013; Kelemen \& Rosset, 2009; Zemla et al., 2012).

650 According to this view, there exist two qualitatively different types of cognitive processes; type-1 processes which are fast, effortless, and do not require working memory, and type-2 processes which are slow, effortful, and do require working memory (e.g., Evans \& Stanovich, 2013). In the current context, teleological beliefs are conceptualised as resulting from a type-1 process. According to this view, as speeded responding precludes the opportunity to engage in reflective thought, responses in the speeded condition are said to reflect a person's belief at an implicit level. Second, it is implicitly assumed that teleological and non-teleological beliefs about a given entity or phenomenon cannot coexist at a prereflective level and be held with equivalent conviction. The finding that response latencies did not differ between teleological statements that were correctly rejected and teleological 
660 statements that were incorrectly accepted, suggests that if this first assumption is true, and

661 teleological beliefs do result from type-1 cognitive processes, the second assumption must be 662 false.

Although non-experimental evidence suggests teleological beliefs are the default

664 when alternative causal modes of explanation have not been obtained through formal

665 education (Casler \& Kelemen, 2008), or when alternative knowledge structures are impaired

666 as a result of neurodegeneration (Lombrozo et al., 2007), this does not speak to whether the non-teleological beliefs of educated individuals without neurological impairment are merely "expressed", or whether they are held at a deeper "implicit" level. Recent work in the field of judgment and decision-making suggests that multiple automatic responses to a given stimulus can occur in parallel (De Neys, 2012, 2014; Pennycook et al., 2015). For example, people are intuitively aware of both the believability (Franssens \& De Neys, 2009) and logical structure of arguments (Morsanyi \& Handley, 2012; Trippas et al., 2016), despite the fact that each of these factors may interfere with judgements of the other factor. People may hold implicit teleological beliefs about biological and nonbiological natural entities, but if they also have an intuitive awareness of an alternative non-teleological explanation, time pressure would not necessarily lead to the expression of the teleological explanation over the alternative. Given the broader field of thinking and reasoning has moved towards dual-process models which allow for multiple type-1 processes to co-occur, theories which situate teleological beliefs with a dual-process framework should do the same. Future research should seek to test alternative paradigms that are not contingent upon the assumption that teleological and nonteleological beliefs about a particular entity cannot coexist implicitly and be held with equivalent conviction. 
either logically valid or invalid, people are often biased by their belief in the conclusion of

686

687

688

689

690

691

692

693

694

695

696

697

698

699

700

701

702

703

704

705

706

707

708 the argument (Pennycook et al., 2013, 2014). Specifically, when a logically valid argument contains an unbelievable conclusion, or when a logically invalid argument contains a believable conclusion, people experience a conflict between logical validity and belief, and are less accurate and slower to respond compared to when the logical validity and believability of the conclusion are congruent (Franssens \& De Neys, 2009; Trémolière et al., 2014). By pairing teleological and non-teleological conclusions with both logically valid and logically invalid argument structures, it would be possible, based on an individual's stated beliefs, to determine whether they should experience a conflict for any given argument. Making use of the belief bias effect in this way, it may be possible to dissociate implicitly held and explicitly expressed teleological beliefs.

Although the current findings may seem inconsistent with the existing literature in showing that the speeded vs. un-speeded paradigm does not provide evidence of a dissociation of implicit and explicit teleological beliefs, many aspects of our work are consistent with the existing literature. First, the bivariate relationships between belief in God, anthropomorphism, inhibition of intuitions, and teleological acceptance replicate previous findings (Roberts et al., 2021; Willard et al., 2020; Willard \& Norenzayan, 2013; Zemla et al., 2012). Second, in terms of the effect of time pressure on teleological test statements, the difference in accuracy between conditions (17\% Study One; $21 \%$ Study Two) are similar to previously reported findings. In fact, the mean difference between conditions reported in previous studies (Kelemen et al., 2013; Roberts et al., 2020) are contained within the confidence intervals of the current results. In conjunction with the fact that the findings of Study One replicated when using a large undergraduate sample in Study Two, this provides additional confidence in the current results. 
In summary, the current studies have shown that the standard experimental paradigm

710 for demonstrating tacit teleological tendencies - to compare rates of teleological acceptance

711 in an un-speeded condition to that in a speeded condition - may not, in fact, provide evidence

712 for such tendencies. Rather, this paradigm results in selection bias and increased rates of

713 acceptance for statements that are objectively false. This is more than just a methodological

714 issue, as the use of speeded decision-making in this context is based on the theoretical

715 assumption that teleological beliefs can be conceptualised within a dual-process framework.

716 We have argued that for the dual-process perspective of teleological beliefs to accommodate

717 the current results, a particular aspect of this view may need to be modified. Teleological

718 beliefs about biological and nonbiological natural entities may well be a "developmentally

719 persistent cognitive default" (Kelemen et al., 2013, p. 1075), but for adults who have learnt

720 alternative mechanistic explanations, there is no reason why teleological beliefs could not co-

721 exist with their non-teleological alternatives at an implicit level. This reframing of the

722 current dual-process perspective of teleology would not only align with recent theoretical and

723 empirical work within the broader field of thinking and reasoning (De Neys, 2012, 2014; De

724 Neys \& Franssens, 2009; Franssens \& De Neys, 2009; Howarth et al., 2016; Morsanyi \&

725 Handley, 2012; Pennycook et al., 2015), but it would also inform new methodologies in the

726 study of teleological beliefs. Speeded decision-making does not reveal tacit teleological

727 tendencies, and this reframing of the dual-process perspective of teleology explains why. 
729

730

731

732

733

734

735

736

737

738

739

740

741

742

743

744

745

746

747

748

749

750

751

752

Atran, S., \& Norenzayan, A. (2004). Religion's evolutionary landscape: Counterintuition, commitment, compassion, communion. Behavioral and Brain Sciences, 27(2004), 1-67.

Boyer, P. (1994). The naturalness of religious ideas: A cognitive theory of religion. University of California Press.

Casler, K., \& Kelemen, D. (2008). Developmental continuity in teleo-functional explanation: Reasoning about nature among Romanian Romani adults. Journal of Cognition and Development, 9(3), 340-362. https://doi.org/10.1080/15248370802248556

De Neys, W. (2012). Bias and conflict: A case for logical intuitions. Perspectives on Psychological Science, 7(1), 28-38. https://doi.org/10.1177/1745691611429354

De Neys, W. (2014). Dual process theory 2.0 (W. De Neys (ed.)). Routledge.

De Neys, W., \& Franssens, S. (2009). Belief inhibition during thinking: Not always winning but at least taking part. Cognition, 113(1), 45-61. https://doi.org/10.1016/j.cognition.2009.07.009

Dennett, D. C. (1987). The intentional stance. MIT Press.

Dennett, D. C. (2017). From bacteria to Bach and back. W. W. Norton \& Company.

Evans, J. S. B. T., \& Stanovich, K. E. (2013). Dual-process theories of higher cognition: Advancing the debate. Perspectives on Psychological Science, 8(3), 223-241. https://doi.org/10.1177/1745691612460685

Fodor, J. (1983). The modularity of mind: An essay on faculty psychology. MIT Press.

Franssens, S., \& De Neys, W. (2009). The effortless nature of conflict detection during thinking. Thinking \& Reasoning, 15(2), 105-128. https://doi.org/10.1080/13546780802711185

Frederick, S. (2005). Cognitive reflection and decision making. Journal of Economic Perspectives, 19(4), 25-42. https://doi.org/10.1257/089533005775196732 
753 Grossi, G. (2014). A module is a module is a module: evolution of modularity in

754

755

756

757

758

759

760

761

762

763

764

765

766

767

768

769

770

771

772

773

774

775

776

777

Evolutionary Psychology. Dialectical Anthropology, 38(3), 333-351. https://doi.org/10.1007/s10624-014-9355-0

Hempel, C. G., \& Oppenheim, P. (1948). Studies in the logic of explanation. Philosophy of Science, 15(2), 135-175.

Howarth, S., Handley, S. J., \& Walsh, C. (2016). The logic-bias effect: The role of effortful processing in the resolution of belief-logic conflict. Memory \& Cognition, 44(2), 330349. https://doi.org/10.3758/s13421-015-0555-x

Huber, S., \& Huber, O. W. (2012). The centrality of religiosity scale (CRS). Religions, 3, 710-724. https://doi.org/10.3390/rel3030710

Järnefelt, E., Canfield, C. F., \& Kelemen, D. (2015). The divided mind of a disbeliever: Intuitive beliefs about nature as purposefully created among different groups of nonreligious adults. Cognition, 140, 72-88. https://doi.org/10.1016/j.cognition.2015.02.005

Kelemen, D. (1999a). Beliefs about purpose: On the origins of teleological thought. In M. C. Corballis \& S. E. G. Lea (Eds.), The descent of mind: Psychological perspectives on hominid evolution (pp. 278-310). Oxford University Press.

Kelemen, D. (1999b). The scope of teleological thinking in preschool children. Cognition, 70(3), 241-272. https://doi.org/10.1016/S0010-0277(99)00010-4

Kelemen, D. (1999c). Why are rocks pointy? Children's preference for teleological explanations of the natural world. Developmental Psychology, 35(6), 1440-1452. https://doi.org/10.1037/0012-1649.35.6.1440

Kelemen, D., \& Diyanni, C. (2005). Intuitions about origins: Purpose and intelligent design in children's reasoning about nature. Journal of Cognition and Development, 6(1), 3-31. https://doi.org/10.1207/s15327647jcd0601

Kelemen, D., \& Rosset, E. (2009). The Human function compunction: Teleological 
explanation in adults. Cognition, 111(1), 138-143. https://doi.org/10.1016/j.cognition.2009.01.001

Kelemen, D., Rottman, J., \& Seston, R. (2013). Professional physical scientists display

781

782

783

784

785

786

787

788

789

790

791

792

793

794

795

796

797

798

799

800

801

802 tenacious teleological tendencies: Purpose-based reasoning as a cognitive default. Journal of Experimental Psychology: General, 142(4), 1074-1083. https://doi.org/10.1037/a0030399

Knowles, E. S., \& Condon, C. A. (1999). Why people say “yes": A dual-process theory of acquiescence. In Journal of Personality and Social Psychology (Vol. 77, Issue 2, pp. 379-386). https://doi.org/10.1037//0022-3514.77.2.379

Kurzban, R. (2010). Why Everyone Else is a Hypocrite: Evolution and the Modular Mind. Princeton University Press.

Lakens, D. (2013). Calculating and reporting effect sizes to facilitate cumulative science: A practical primer for t-tests and ANOVAs. Frontiers in Psychology, 4(NOV), 1-12. https://doi.org/10.3389/fpsyg.2013.00863

Liquin, E. G., \& Lombrozo, T. (2018). Structure-function fit underlies the evaluation of teleological explanations. Cognitive Psychology, 107(October), 22-43. https://doi.org/10.1016/j.cogpsych.2018.09.001

Lombrozo, T., \& Carey, S. (2006). Functional explanation and the function of explanation. Cognition, 99(2), 167-204. https://doi.org/10.1016/j.cognition.2004.12.009

Lombrozo, T., Kelemen, D., \& Zaitchik, D. (2007). Inferring design: Evidence for a preference for teleological explanations in patients with Alzheimer's disease. Psychological Science, 18(11), 999-1007.

Mills, R., \& Frowley, J. (2014). Promiscuous Teleology and the effect of Locus of Control. Irish Journal of Psychology, 35(2-3), 121-132. https://doi.org/10.1080/03033910.2015.1011192 
803 Morsanyi, K., \& Handley, S. J. (2012). Logic feels so good-I like it! evidence for intuitive detection of logicality in syllogistic reasoning. Journal of Experimental Psychology: Learning Memory and Cognition, 38(3), 596-616. https://doi.org/10.1037/a0026099

Neave, N., Jackson, R., Saxton, T., \& Hönekopp, J. (2015). The influence of anthropomorphic tendencies on human hoarding behaviours. Personality and Individual

Pennycook, G., Cheyne, J. A., Barr, N., Koehler, D. J., \& Fugelsang, J. A. (2014). Cognitive style and religiosity: The role of conflict detection. Memory \& Cognition, 42(1), 1-10. https://doi.org/10.3758/s13421-013-0340-7

Pennycook, G., Cheyne, J. A., Koehler, D. J., \& Fugelsang, J. A. (2013). Belief bias during reasoning among religious believers and skeptics. Psychonomic Bulletin \& Review, 20(4), 806-811. https://doi.org/10.3758/s13423-013-0394-3

Pennycook, G., Fugelsang, J. A., \& Koehler, D. J. (2015). What makes us think? A threestage dual-process model of analytic engagement. Cognitive Psychology, 80, 34-72. https://doi.org/10.1016/j.cogpsych.2015.05.001

Purcell, Z. A., Wastell, C. A., \& Sweller, N. (2020). Domain-specific experience and dualprocess thinking. Thinking and Reasoning, $0(0), 1-29$. https://doi.org/10.1080/13546783.2020.1793813

Roberts, A. J., Handley, S. J., \& Polito, V. (2021). The design stance, intentional stance, and teleological beliefs about biological and non-biological natural entites. Journal of Personality and Social Psychology, 120(6), 1720-1748. https://doi.org/10.1037/pspp0000383

Roberts, A. J., Wastell, C. A., \& Polito, V. (2020). Teleology and the intentions of supernatural agents. Consciousness and Cognition, 80(February), 102905. 
828 Rottman, J., Zhu, L., Wang, W., Seston Schillaci, R., Clark, K. J., \& Kelemen, D. (2017).

829 Cultural influences on the teleological stance: evidence from China. Religion, Brain \& 830 Behavior, 7(1), 17-26. https://doi.org/10.1080/2153599X.2015.1118402

831 Thomson, K. S., \& Oppenheimer, D. M. (2016). Investigating an alternate form of the 832 cognitive reflection test. Judgment and Decision Making, 11(1), 99-113. 833 https://doi.org/ISBN 978-615-5270-17-8

834 Trémolière, B., De Neys, W., \& Bonnefon, J. F. (2014). The grim reasoner: Analytical 835 reasoning under mortality salience. Thinking and Reasoning, 20(3), 333-351. 836 https://doi.org/10.1080/13546783.2013.823888

837 Trippas, D., Handley, S. J., Verde, M. F., \& Morsanyi, K. (2016). Logic brightens my day:

$838 \quad$ Evidence for implicit sensitivity to logical validity. Journal of Experimental

839 Psychology: Learning Memory and Cognition, 42(9), 1448-1457. https://doi.org/10.1037/xlm0000248

841 White, C. J., Baimel, A., \& Norenzayan, A. (2021). How cultural learning and cognitive 842 biases shape religious beliefs. Current Opinion in Psychology, 40, 34-39.

843 https://doi.org/10.1016/j.copsyc.2020.07.033

844 Willard, A. K., Cingl, L., \& Norenzayan, A. (2020). Cognitive Biases and Religious Belief: A 845 Path Model Replication in the Czech Republic and Slovakia With a Focus on 846 Anthropomorphism. Social Psychological and Personality Science, 11(1), 97-106.

847 https://doi.org/10.1177/1948550619841629

848 Willard, A. K., \& Norenzayan, A. (2013). Cognitive biases explain religious belief, 849 paranormal belief, and belief in life's purpose. Cognition, 129(2), 379-391. $850 \quad$ https://doi.org/10.1016/j.cognition.2013.07.016

851 Wright, L. (1976). Teleological explanations. University of California Press.

852 Zemla, J. C., Steiner, S. M., \& Sloman, S. (2012). Analytical Thinking Predicts Less 

of the Cognitive Science Society, August, 1217-1222. 